\title{
Haben Sie den Überblick?
}

\section{Wissen Sie, welche Leistungen Ihnen im Vorsorgefall zustehen?}

In den letzten 60 Jahren wurde unser Vorsorgesystem Schritt für Schritt aufgebaut und basiert heute auf dem bekannten 3-SäulenKonzept. Durch diesen kontinuierlichen Ausbau hat das gesamte System eine enorme Komplexität angenommen. Oftmals sind Leistungen abhängig von der Ursache (Krankheit, Unfall) und nicht von der entsprechenden Wirkung (z. B. Lohnausfall bei Erwerbsunfähigkeit). Der Überblick kann dadurch schnell verloren gehen.

Nachfolgend sehen Sie eine kurze Checkliste, um zu überprüfen, ob Sie über die Vorsorgesituation Ihrer Familie Bescheid wissen:

\begin{tabular}{|c|c|c|}
\hline Vorsorgefall & $\begin{array}{l}\text { Mögliche Leistungserbringer } \\
\text { (nicht abschliessend) }\end{array}$ & $\begin{array}{l}\text { Kennen Sie lhre } \\
\text { Leistungen? }\end{array}$ \\
\hline $\begin{array}{l}\text { Todesfall } \\
\text { (infolge Krankheit) }\end{array}$ & AHV, Pensionskasse, private Versicherungen & $O$ JA O NEIN \\
\hline $\begin{array}{l}\text { Todesfall } \\
\text { (infolge Unfall) }\end{array}$ & AHV, UVG, Pensionskasse, private Versicherungen & $\bigcirc$ JA O NEIN \\
\hline $\begin{array}{l}\text { Erwerbsunfähigkeit } \\
\text { (infolge Krankheit) }\end{array}$ & $\begin{array}{l}\text { Lohnfortzahlung des Arbeitgebers, IV, Krankentaggeld, } \\
\text { Pensionskasse, private Versicherungen }\end{array}$ & $\bigcirc$ JA $\bigcirc$ NEIN \\
\hline $\begin{array}{l}\text { Erwerbsunfähigkeit } \\
\text { (infolge Unfall) }\end{array}$ & $\begin{array}{l}\text { Lohnfortzahlung des Arbeitgebers, IV, UVG, Pensionskasse, } \\
\text { private Versicherungen }\end{array}$ & O JA O NEIN \\
\hline Pensionierung & AHV, Pensionskasse, private Vorsorge & $\bigcirc \mathrm{JA} \bigcirc \mathrm{NEIN}$ \\
\hline Invalidität der Kinder & IV, Ergänzungsleistungen, private Versicherungen & O JA O NEIN \\
\hline
\end{tabular}

\section{Auswertung}

$0 \times$ NEIN: Herzlichen Glückwunsch, Sie scheinen Ihre Vorsorgesituation bestens zu kennen. Gerne können wir aber überprüfen, ob Sie bereits von den besten Lösungen profitieren.

1-6 × NEIN: Wir empfehlen Ihnen, Ihre Vorsorgesituation durch einen FMH-Insurance-Services-Spezialisten überprüfen zu lassen. In einer Analyse definieren Sie Ihren Vorsorgebedarf (SOLL-Situation). Unsere Berater zeigen Ihnen auf, wo Sie sich heute befinden (IST-Situation) und wie Sie allfällige Über- oder Unterversicherungen vermeiden können.

\section{Antworttalon}

Vorname / Name

Adresse

PLZ / Ort

Geburtsdatum

Telefon Privat/Geschäft

Beste Zeit für einen Anruf

E-Mail-Adresse

Bitte nehmen Sie mit mir für eine Analyse meiner Vorsorgesituation Kontakt auf.

Ich wünsche eine persönliche Beratung. Bitte rufen Sie mich an.

Ich interessiere mich für:
Säule $3 a$
Pensionskasse BVG
Finanz-/Steuerplanung
Sichere Kapitalanlage
Rechtsschutzversicherung
O
O Berufshaftpflichtversicherung

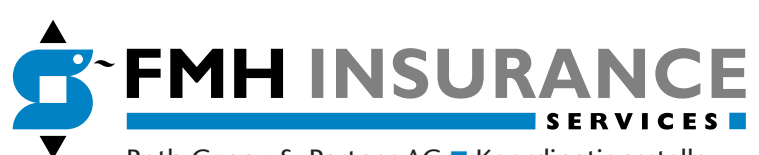

Roth Gygax \& Partner AG a Koordinationsstelle Moosstrasse 2 ॠ 3073 Gümligen

Telefon 0319595000 \&ax 0319595010 mail@fmhinsurance.ch $n$ www.fmhinsurance.ch 